\title{
Microfluidic generation of Buddha beads-like microcarriers for cell culture
}

\author{
Jie Wang, Minhan Zou, Lingyu Sun, Yao Cheng, Luoran Shang, Fanfan Fu and Yuanjin Zhao*
}

\begin{abstract}
The fabrication of functional microcarriers capable of achieving in vivo-like three-dimensional cell culture is important for many tissue engineering applications. Here, inspired by the structure of Buddha beads, which are generally composed of moveable beads strung on a rope, we present novel cell microcarriers with controllable macropores and heterogeneous microstructures by using a capillary array microfluidic technology. Microfibers with a string of moveable and releasable microcarriers could be achieved by an immediate gelation reaction of sodium alginate spinning and subsequent polymerization of cell-dispersed gelatin methacrylate emulsification. The sizes of the microcarriers and their inner macropores could be well tailored by adjusting the flow rates of the microfluidic phases; this was of great importance in guaranteeing a sufficient supply of nutrients during cell culture. In addition, by infusing multiple cell-dispersed pregel solutions into the capillaries, the microcarriers with spatially heterogeneous cell encapsulations for mimicking physiological structures and functions could also be achieved.
\end{abstract}

Keywords: microfluidics, microcarrier, cell culture, microfiber, emulsion

\section{INTRODUCTION}

Recent technological advances in engineering three dimensional (3D) cell cultures have demonstrated prominent improvement in approximation of cell-cell interactions and microenvironmental conditions in vivo, which play a great role in the field of tissue engineering [1-11]. Among them, microcarriers have emerged as novel biomimetic platforms, which offer 3D biomaterial scaffolds for cell encapsulation and aggregate formation [10-15]. Several approaches have been proposed for fabricating microcarriers, such as photolithography, micromolding, electrojetting and microfluidics [16-24]. In comparison with other approaches, microfluidic micro- carriers are a promising technique for cell encapsulation because of their advantages of excellent monodispersity, precise size control, high throughput, and better microenvironmental control [21-28]. However, because of the lack of effective nutrient exchange pathways, most of the microcarriers are restricted to a small size to avoid the formation of necrotic regions [29-31]. In addition, conventional microcarriers could only provide a homogeneous microenvironment for uniform cell distribution, whereas cell distribution in vivo is extremely complex. Spatially anisotropic or heterogeneous tissues are widespread, which is of great significance in maintaining the physiological functions of organisms. Microcarriers with heterogeneous structures are aimed at paving the way for mimicking complex organisms in vitro [2,32,33]. Thus, novel microcarriers with more sophisticated structures and functions, and sufficient supply of oxygen and nutrients are still anticipated.

In this paper, inspired by the flexible structure of Buddha beads, which are generally composed of moveable beads strung on a rope (Scheme 1a), we present novel cell microcarriers with controllable macropores and heterogeneous microstructures based on a capillary array microfluidic technology, as illustrated in Scheme 1b. With an immediate gelation reaction of sodium alginate $(\mathrm{Na}-$ Alg) spinning solution from the central capillary and subsequent polymerization of cell-dispersed gelatin methacrylate (GelMA) emulsions from the surrounding capillaries, a microfiber with a string of microcarriers could be achieved. Similar to the Buddha beads, the microcarriers could be peeled off from the microfibers because of their moveable property, which resulted from different components and viscosities of the alginate and GelMA. By adjusting the flow rates of the microfluidic phases, the sizes of the microfibers and emulsions are highly tunable, resulting in microcarriers with controllable macropore

\footnotetext{
State Key Laboratory of Bioelectronics, School of Biological Science and Medical Engineering, Southeast University, Nanjing 210096, China

* Corresponding author (email: yjzhao@seu.edu.cn)
} 


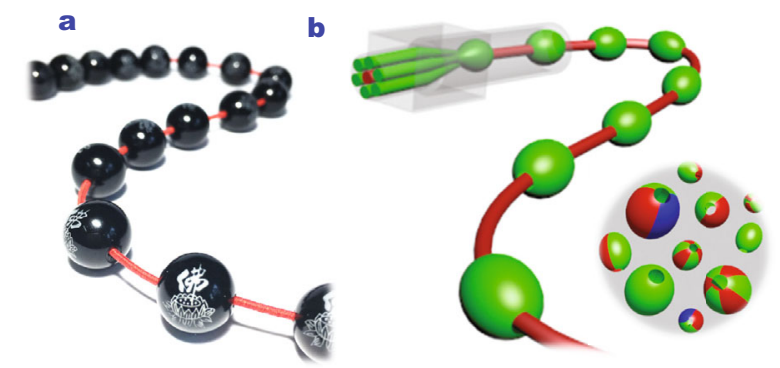

Scheme 1 Schematic diagram of (a) the Buddha beads and (b) the capillary array microfluidic technology for the generation of coaxial macropore microcarriers.

sizes, which is a very important feature for guaranteeing sufficient supply of nutrients during cell culture. Moreover, by infusing multiple cell-dispersed pregel solutions into the surrounding capillaries, the microcarriers with spatially anisotropic or heterogeneous cell encapsulations could also be achieved for mimicking physiological structures and functions. These features make the microcarriers described here ideal for applications in tissue engineering.

\section{EXPERIMENTAL SECTION}

\section{Materials}

Materials for the inner phase of the microfluidics were 3\% $(w / v)$ sodium alginate (Na-alginate, very low viscosity, Alfa Aesar). The middle phase was a mixture solution of $35 \%(w / v)$ methacrylated gelatin (GelMA, self-synthesized), $2 \%(w / v)$ calcium chloride $\left(\mathrm{CaCl}_{2}\right.$, Anhydrous, Alfa Aesar) and 1\% $v / v$ photoinitiator (2-hydroxy-2-methylpropiophenone, Sigma, USA). Silicone oil (Shinetsu, $100 \mathrm{cs}$, Japan) was used as the outer phase. 1\% $(v / v)$ fluorescent polystyrene nanoparticles (Invitrogen: F8805 (excitation/emission: 365/415 nm), F8810 (580/605) and F8811 (505/515)) were also added in the middle phases for confocal laser scanning microscopy (CLSM) images demonstrating multicompartment of the microcarriers. Solutions were all filtered before pumped into glass microcapillary devices. Water with a resistivity of $18.2 \mathrm{M} \Omega$ $\mathrm{cm}^{-1}$ was acquired from a Millipore Milli-Q system. All other chemical reagents were of the best grade available and used as received.

\section{Microfluidics}

The capillary microfluidic device consists of coaxial assemblies of glass capillaries with different shapes and models (World Precision Instruments) on glass slides. A seven-barrel capillary array (outer/inner diameter of every capillary: $0.68 / 0.58 \mathrm{~mm}$ ) was tapered to the desired orifice by a capillary puller (Sutter Instrument, P-97) and a microforge (Narishige, MF-830). The tapered capillary array was inserted into a cylindrical capillary (outer/inner diameter: $1 / 0.58 \mathrm{~mm})$. Both of them were inside of a square capillary (VitroCom, Inc.) with an inner diameter of $1.05 \mathrm{~mm}$. The connectors of the assembled capillaries were sealed with dispensing needles and transparent epoxy resin (Devcon 5 Minute Epoxy) where necessary. All fluids were pumped into the capillary microfluidic devices by syringe pumps (Harvard PHD 2000 series). The resulting microfibers were polymerized in the collection channel by a UV light $(365 \mathrm{~nm}, \mathrm{~S} 1000$, OmniCure, Mississauga, Ontario, Canada) with the photoinitiator (2hydroxy-2-methylpropiophenone, 1 vol\%, Sigma, USA) at the distance of 5 to $10 \mathrm{~cm}$ for 5 to $10 \mathrm{~s}$ and then were collected in glass vials that were filled with deionized water.

\section{Cell encapsulations and culture}

HepG2 cells and NIH-3T3 cells (obtained from the Cell Bank of the Chinese Academy of Sciences, Shanghai, China) were homogeneously dispersed in the GelMA solution at a concentration of $3 \times 10^{6}$ cells $/ \mathrm{mL}$. All the cells were incubated with sufficient culture medium in the incubator (HERA cell 150, Thermo, USA) with 5\% $\mathrm{CO}_{2}$ at $37^{\circ} \mathrm{C}$. The culture medium was a high sugar Dulbecco's modified Eagle's medium (High Glucose DMEM, Invitrogen, Nanjing and Biotech Development Co. Ltd) supplemented with $10 \%$ fetal bovine serum, $1 \% 10,000$ $\mathrm{U} \mathrm{mL}^{-1}$ penicillin and $10,000 \mu \mathrm{g} \mathrm{mL}^{-1}$ streptomycin (Gibco, Life Technolegy). During the culture, the cells were stained by $10 \mu \mathrm{mol} \mathrm{L}{ }^{-1}$ Calcein-AM (Molecular Probes Co.) and propidium iodide (PI, Molecular Probes Co.) for observation. 3-(4,5-dimethylthiazol-2-yl)-2,5-diphenyltetrazolium bromide (MTT, 98\%, J\&K Scientific Ltd., Shanghai) was used for quantitative analysis of cell viability. The number of replicates was 6 . For the investigation of the co-cultured cells, HepG2 and NIH-3T3 cells were stained by 3,3-dioctadecyloxa-carbocyanineperchlorate (DIO, a green fluorescent dye) and 1,1-dioctadecyl-3,3,3,3-tetramethylindodicarbocyanine (DiD, a red fluorescent dye) cell-labeling solutions (Invitrogen Co.), respectively, before the encapsulation. MTT was used for quantitative analysis of cell viability of the cocultured microcarriers. The number of replicates was 7 . The albumin secretion was measured using the Rat Albumin ELISA Quantitation kit (Bethyl Laboratories Inc., Montgomery, USA). The urea synthesis was detected with the Urea Nitrogen kit (Biochain Institute Inc., Boerne, 
USA). The number of replicates for each group was 4. All processes of the testing follow the provided kit protocol completely.

\section{Characterization}

The microcarrier generating processes in our capillary microfluidic device were monitored using a microscope (SMZ 745T, Nikon, Tianjin, China) fitted with a fast camera (S-PRI F1, AOS Technologies AG). Optical microscope images of the microcarriers were obtained with a stereo microscope (MVX10, Olympus, Tokyo, Japan) and recorded by a charged coupled device (CCD, Media Cybernetics Evolution MP 5.0 RTV). Fluorescence images of the microcarriers were obtained by an inverted fluorescence microscope (IX71, Olympus, Tokyo, Japan) equipped with CCD cameras (DP30BW, Olympus, Tokyo, Japan). Morphology features of the microcarriers were explored by scanning electron microscopy (SEM) images using a field emission scanning electron microscope (FESEM, Ultra Plus, Zeiss). CLSM images of microcarriers were taken by a confocal laser scanning microscopy (FV10i, Olympus, Tokyo, Japan).

\section{Statistical analyses}

The significance of the differences between groups was tested by one-way analysis of variance (ANOVA) followed by the least significant difference (LSD) test using the IBM SPSS Statistics 17.0 software (IBM Japan, Tokyo, Japan). A probability value of $p<0.05$ was considered statistically significant.

\section{RESULTS AND DISCUSSION}

In a typical experiment, the microfluidic device was constructed by aligning a tapered annular capillary array and a collection capillary coaxially located inside a square capillary. The annular capillary array consisted of seven identical cylindrical glass capillaries with inner and outer diameters of 0.58 and $0.68 \mathrm{~mm}$, respectively. The capillary array could be tapered uniformly to desired lengths and diameters, with the orifice maintaining the same structure as the original capillary array. A square glass with an inner diameter of $1.05 \mathrm{~mm}$ was used in the assembly of the tapered capillary array and inside the collection capillary to integrate fluids conveniently into the collection capillary channel and observe the process of emulsification.

The inner phase of Na-Alg was pumped through the central capillary of the seven-barrel capillary array. The middle phase was the mixture of GelMA and $\mathrm{CaCl}_{2}$ solution that flowed through the six surrounding capillaries.
Silicone oil solution was pumped in the same direction through the region between the inner capillaries and the outer square capillary as the outer phase. Because of the fast diffusion that controlled the ion cross-linking of $\mathrm{Na}$ Alg and $\mathrm{CaCl}_{2}$, a calcium alginate (Ca-Alg) hydrogel microfiber was generated in situ, spun continuously, and then coated by the photocurable GelMA solution (Fig. 1a). Once flowed into the immiscible oil solution, the coating GelMA solution was emulsified and broke into droplets which were strung along the $\mathrm{Ca}$-Alg microfiber. The shear force of the oil solution pushed the GelMA droplets forward, while the interface tension of the GelMA solution tended to pull them back. When the shear force of the outer oil phase and the interface tension of the middle phase reached equilibrium, the water/oil interface deformed and spindle-shaped droplets coated microfibers could be achieved. By adjusting the flow rates of different streams, the size of the droplets and the fibers were highly controllable, as investigated in Fig. 1. The size of the droplets showed a negative association with the inner $\left(F_{1}\right)$ and outer flow rate $\left(F_{3}\right)$, and a positive association with the middle flow rate $\left(F_{2}\right)$ (red points in Fig. $1 \mathrm{~b}-\mathrm{d}$ ); while the size of the microfibers showed a positive association with $F_{1}$ and a negative association with $F_{3}$ (blue points in Fig. 1b, d). These results indicate that the sizes of the droplets and the microfibers could be quantitatively controlled through changing the flow rates of the three streams, which would be very important in the size control of the resulting microcarriers.

Solidified Ca-Alg microfibers with GelMA microcarrier coating could be continuously generated by exposing the collection capillary to ultraviolet (UV) irradiation for polymerizing the GelMA pre-gel droplets on the microfiber surfaces, as shown in Fig. 2. It was found that the components of the polymerized GelMA microcarriers were independent from their strung microfibers, as confirmed by CLSM images (Fig. $2 \mathrm{a}-\mathrm{c}$ ) and the microstructure presented in the SEM images (Fig. 2d). The inner alginate fibers and the surrounding GelMA microcarriers were all polymerized very fast, and they could not diffuse to each other, thus they were not strongly connected. This should be ascribed to the difference in components, chemical surface and viscosities of the CaAlg and GelMA hydrogels. Thus, the solidified microcarriers had free movement along the microfibers and could be peeled off from the strung microfibers like Buddha beads, as shown in Fig. 2e, $\mathrm{f}$ and Movie S1. To ensure the success of this process, a relatively high concentration of $\mathrm{Na}$-Alg should be employed to make the microfibers robust and easy to be extracted from the 
$\mathbf{a}$
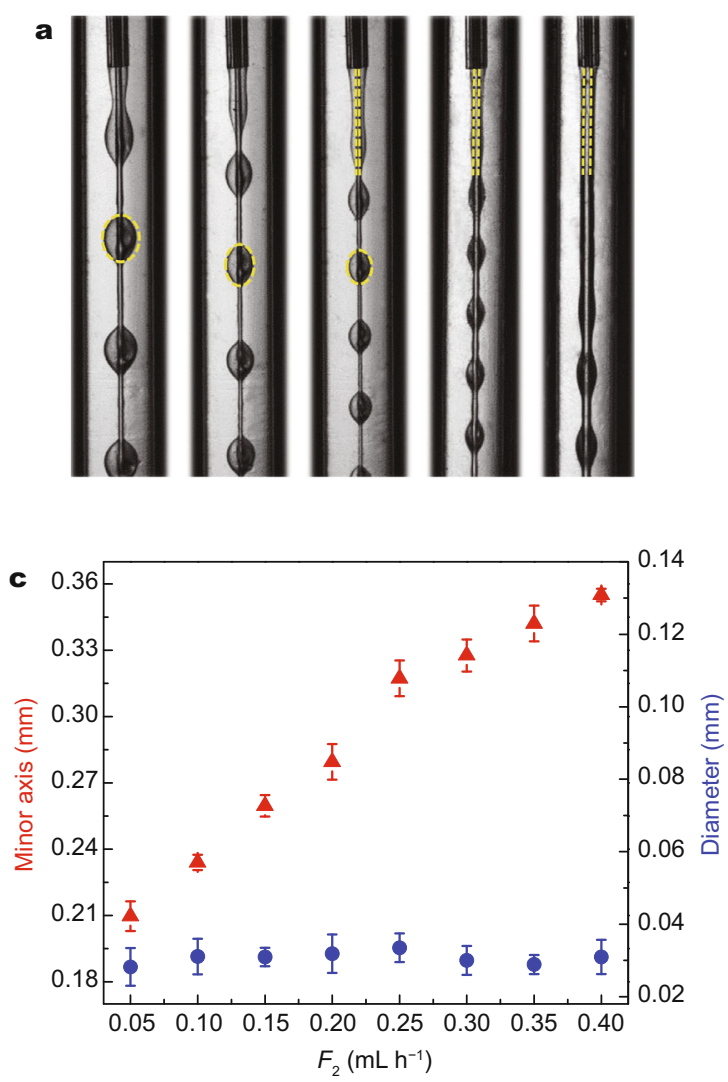
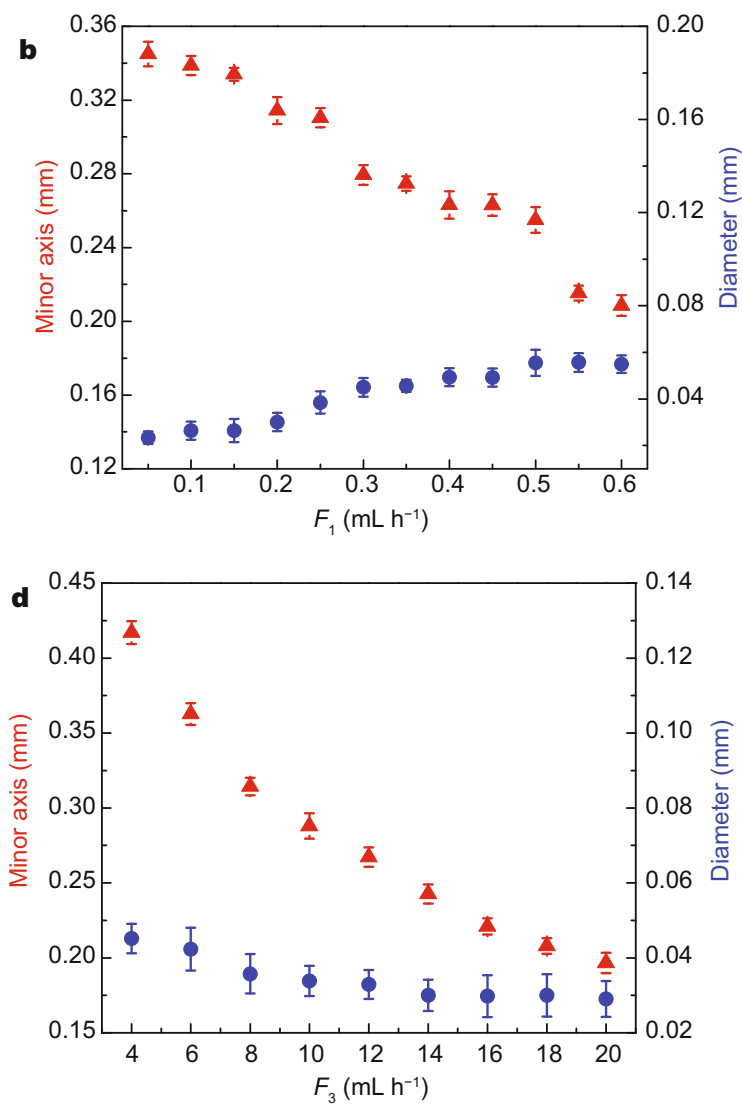

Figure 1 (a) Real-time microscopic images of the microfluidic generation process of the droplets coated microfibers with tunable sizes of droplets and microfibers. (b-d) Relationships between the minor axis of the droplets (red color), the diameter of microfibers (blue color) and flow rates of three streams $\left(F_{1}, F_{2}, F_{3}\right) . F_{2}$ was $0.2 \mathrm{~mL} \mathrm{~h}^{-1}$ and $F_{3}$ was $10 \mathrm{~mL} \mathrm{~h}^{-1}$ in (b). $F_{1}$ was $0.2 \mathrm{~mL} \mathrm{~h}^{-1}$ and $F_{3}$ was $10 \mathrm{~mL} \mathrm{~h}^{-1}$ in $(\mathrm{c}) . F_{1}$ was $0.2 \mathrm{~mL} \mathrm{~h}^{-1}$ and $F_{2}$ was $0.2 \mathrm{~mL} \mathrm{~h}^{-1}$ in $(\mathrm{d})$.

microcarriers. Besides, the microfibers could also be digested by the alginate lyase in case the low-concentration Na-Alg should be applied.

After removing the strung microfibers, the microcarriers with a single coaxially through macropore could be collected, as shown in Fig. 3. The results indicated that the removal of the microfibers did not destroy the structure of the microcarriers. The size of the macropore in microcarriers remained almost the same as the diameter of the microfibers. Thus, microcarriers with highly controllable size and structure could be constructed. To guarantee sufficient supply of oxygen and nutrients during cell culture, GelMA microcarriers with a minor axis of about $200 \mu \mathrm{m}$, and with the macropores of about $20 \mu \mathrm{m}$ were fabricated in the following experiment.

A noticeable advantage of our method was that unprecedented microcarriers with spatially anisotropic or heterogeneous components could be exploited, as schemed in Fig. 4. During this process, different pregel solutions mixed with different dyes or cells were simultaneously infused into the surrounding capillaries of the microfluidics using the same flow rate. Because of their low Reynolds number, these flow solutions mainly formed laminar flows around the center microfiber and no obvious diffusive mixing occurred across the different color streams before solidification. When these laminar flows were emulsified into droplets on the microfiber, a fast UV polymerization was employed to immobilize the inner structures of the droplet templates (Fig. S1 and Movie S2). From the CLSM images, it could be observed that the microcarrier coated microfibers had nearly axisymmetrical geometries, which should be attributed to the regular capillary array geometries, low disturbance in the microfluidic channels, and the fast solidification of the templates.

The multicompartmental microcarriers with macropores could also be obtained after the same collection and microfiber removal processes as the above simple gen- 


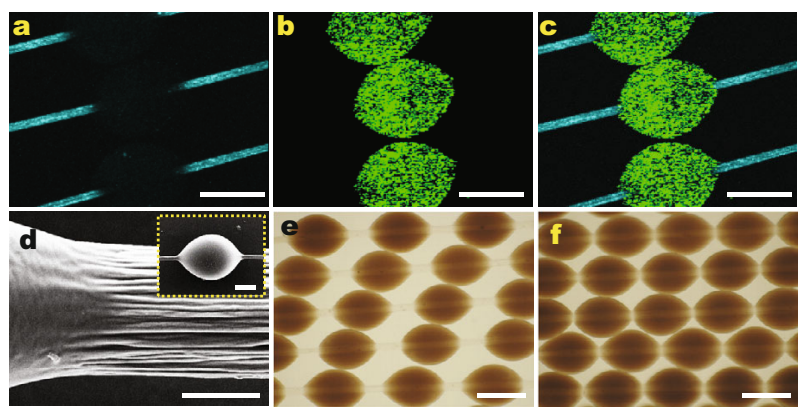

Figure 2 (a-c) CLSM images and (d) SEM images of the microcarriers coated microfibers showing the hetero-structure of the microcarriers and the microfibers. (e, f) Optical microscope images of the same microcarriers coated microfibers before and after stacking the microcarriers, indicating free movement of the microcarriers along microfibers. The scale bars are $200 \mu \mathrm{m}$ in $(\mathrm{a}-\mathrm{c}), 10 \mu \mathrm{m}$ in (d), $100 \mu \mathrm{m}$ in the insert image of $(d)$, and $300 \mu \mathrm{m}$ in (e, f).

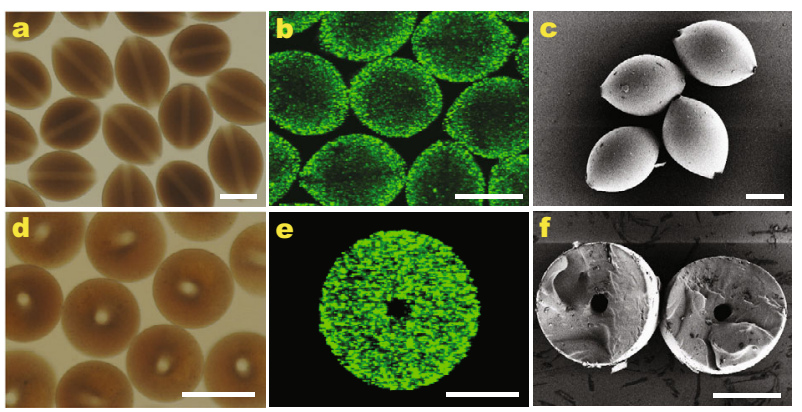

Figure $3(\mathrm{a}-\mathrm{c})$ Optical microscope, CLSM and SEM images of the microcarriers with a single coaxially through macropore; (d-f) cross sections of the corresponding microcarriers in $(\mathrm{a}-\mathrm{c})$. The scale bars are $200 \mu \mathrm{m}$ in $(\mathrm{a}, \mathrm{b}, \mathrm{d})$ and $100 \mu \mathrm{m}$ in $(\mathrm{c}, \mathrm{e}, \mathrm{f})$. a

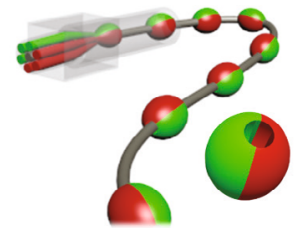

b

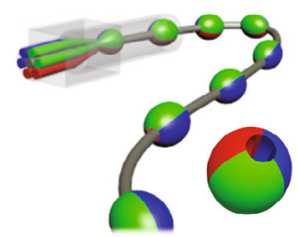

c

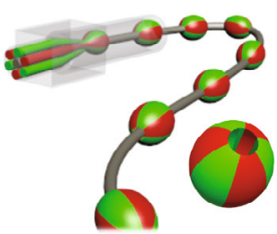

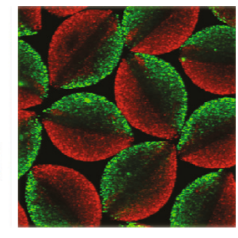
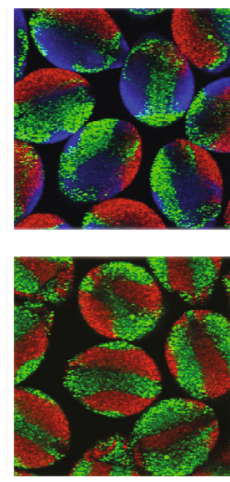
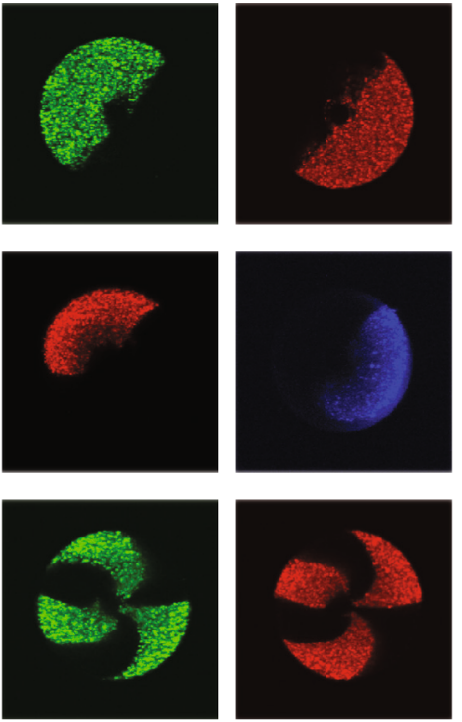
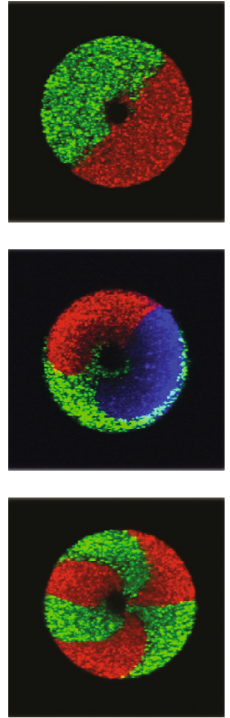

Figure 4 Schematic illustrations and the CLSM images (lateral views in second column, and cross sections in third, fourth and fifth columns) of microfluidic generation of multicompartmental microcarriers: (a) Janus microcarriers; (b) three-compartmental microcarriers; (c) six-compartmental microcarriers.

eration of microcarriers (Fig. S2 and Movie S3). Fig. 4a presents typical cross-sectional CLSM images of the Janus macropore microcarriers. The internal architecture of the microcarriers displayed an extremely sharp boundary at the interface between the two compartments, which suggested minimal mass transfer between the compartments. By extending the two different fluids to three or six different pregel solutions into the surrounding injection capillaries of the microfluidics, three- and six-compartmental microcarriers could correspondingly be generated, as shown in Fig. 4b, c. Each compartment of the microcarriers could have its own chemical composition, which is determined by the chemical composition of the initial injecting solutions. Thus, the multicompartmental microcarriers have important potential applications in creating anisotropic or heterogeneous cell encapsulations and coculture.

To confirm the value of our microcarriers in biomedical engineering, we employed them as controllable cell encapsulations in $3 \mathrm{D}$ cell culture. For this purpose, HepG2 cells were dispersed in GelMA solutions, which was beneficial in reconstructing a microenvironment typical for cell-cell connections due to the presence of a cell attachment site on GelMA molecules. It is worth men- 

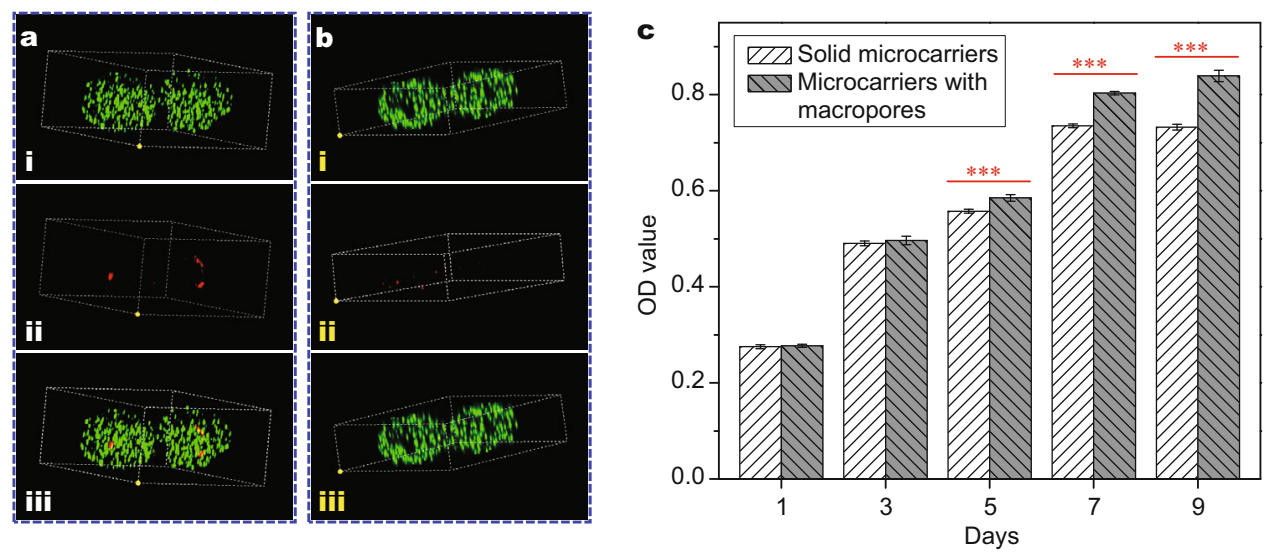

Figure 5 CLSM images of (a) two solid cell microcarriers and (b) two macropore cell microcarriers after culturing for seven days. Dead cells were stained red while living cells were stained green. (c) MTT assays of the cells cultured in microcarriers with different structures. Results are presented as mean value \pm standard error. ${ }^{* * *} p<0.001$.

tioning that no surfactant is needed during the generation of the microcarriers, which would impart the system with less toxicity to cells. In order to demonstrate the superiority of the macropore structure, the macropore microcarriers were comparable with solid microcarriers with the same volume size, material component and quantities of cell concentrations of our microcarriers with macropores, by simply blocking the inner capillary of the same microfluidic device and change $F_{1}$ to zero with the same flow rates of $F_{2}$ and $F_{3}$. Solid microcarriers were found to begin to form necrotic regions inside while few dead cells appeared in the macropore microcarriers after culturing for seven days (Fig. 5a, b). The cell viabilities on these two kinds of microcarriers were also investigated by using MTT assay, which is the most common method of quantitative study of cell viability. It was noticeable that the cells in solid microcarriers almost hardly proliferated after 7 days, while the cells in microcarriers with macropores reached their plateau of growth much later. With the proliferation of the cells, the cells encapsulated in solid microcarriers were found to suffer from insufficient oxygen and nutrients earlier than those on macropore microcarriers. These results indicate the outstanding structure preponderance of the macropore microcarriers for cell encapsulation and culture.

Cell coculture experiments were also investigated in the microcarriers. For this purpose, the HepG2 cells and NIH-3T3 cells dispersed in GelMA solutions were pumped into microfluidic devices to fabricate cell-laden microcarriers with different structures and cell distributions, as shown in Fig. 6a-d. To observe the distribution of cells in the microfibers, the two kinds of cells were stained green and red, respectively, before the en- capsulation. From the CLSM images of the resultant cellladen microcarriers, a distinct interface could be observed at the interface between the HepG2 and NIH-3T3 cells (Fig. S3), which was beneficial for their liver-specific functions. MTT assays were also conducted to show the good cell viabilities in the microcarriers (Fig. S4). To confirm the liver-specific functions of the microcarriers, a Rat Albumin ELISA Quantitation kit and a Urea Nitrogen kit were used to quantify albumin secretion and urea synthesis, as these are two primary bioactive indices of hepatic cells [34]. Compared to the monotypic culture of hepatocytes alone, the coculture of hepatocytes and fibroblasts in the Janus-compartmental microcarriers or in other type of compartmental microcarriers were all found to display both increased albumin secretion and urea synthesis, as shown in Fig. $6 \mathrm{e}, \mathrm{f}$. These results indicate that the coculture of hepatocytes and fibroblasts embedded in our microcarriers is a good model of the liver in vitro. Based on our method, more types of cells or biological factors could be encapsulated inside the compartments of the microcarriers but remained separated from each other. This would make the macropore microcarriers a promising 3D platform for studying interactions between different cell types and constructing complex multi-tissues.

\section{CONCLUSIONS}

In summary, we have presented a capillary array microfluidic technology for continuously spinning Buddha bead-like microfibers with a string of moveable macropore microcarriers. Similar to the Buddha beads, these microcarriers could be peeled off from the strung microfibers because of their movability. The sizes of the 

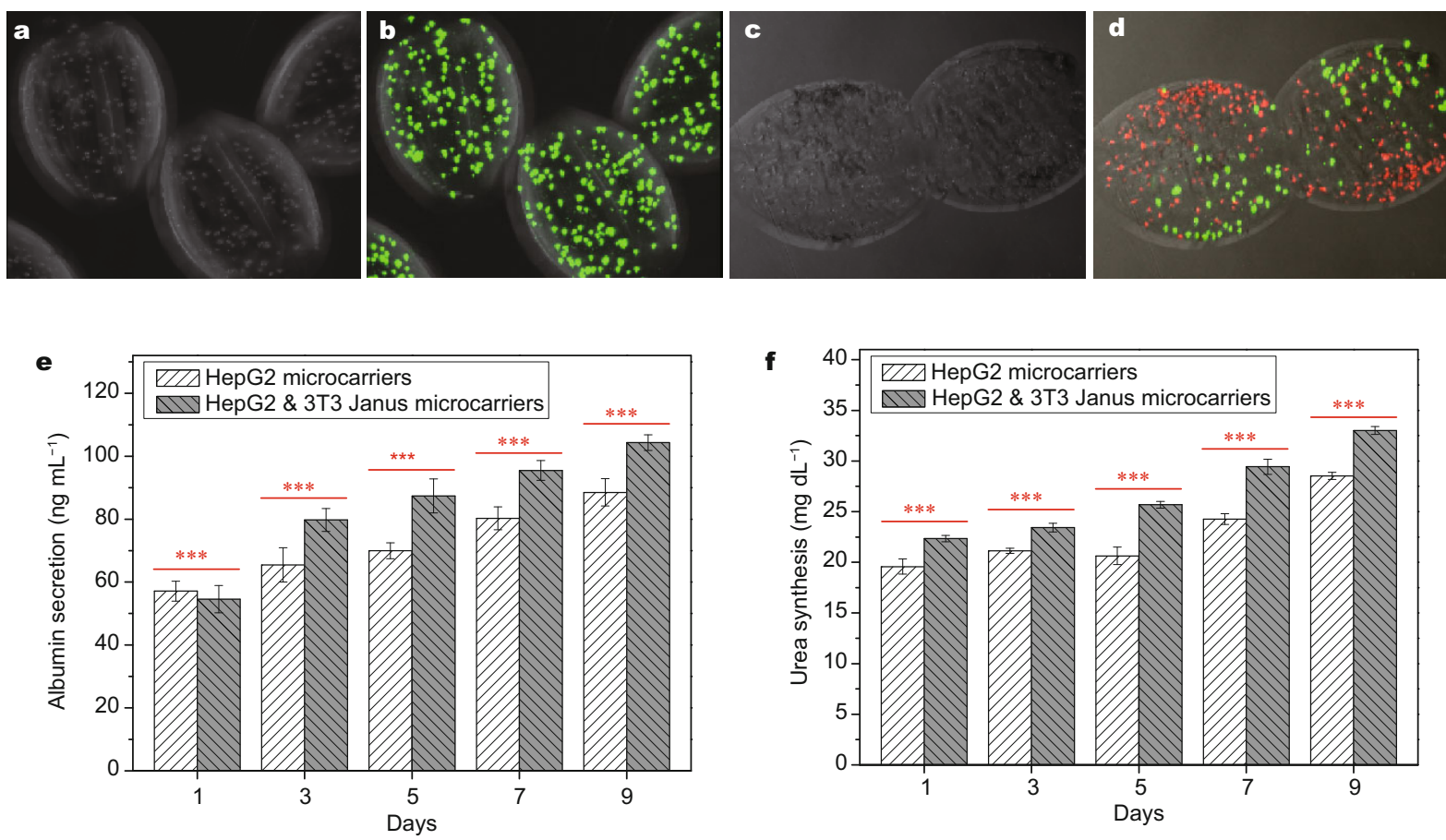

Figure 6 (a-d) Optical images and fluorescent images of one- and two-compartemtal macropore microcarriers. The HepG2 cells were stained with a green fluorescent dye, and NIH-3T3 cells were stained with a red fluorescent dye. (e) Albumin secretion and (f) urea synthesis of the cells in the microcarriers were measured at different time points during a 9-day culture. All dates were measured at the same initial cell number and were expressed as mean value \pm standard error. ${ }^{* *} p<0.001$. The numbers of the HepG2 cells encapsulated in monotypic hepatocyte microcarriers and Janus-compartmental microcarriers are the same. The HepG2 cells and NIH-3T3 cells in (e, f) have the same quantities.

microcarriers and their inner macropore could be well tailored by adjusting the flow rates of the microfluidic injection fluids; this guaranteed the sufficient supply of oxygen and nutrients during cell encapsulation and culture. In addition, by infusing multiple cell-dispersed pregel solutions into the surrounding injection capillaries of the microfluidics, macropore microcarriers with spatially anisotropic or heterogeneous cell encapsulations could also be achieved. The coculture of hepatocytes and fibroblasts in these microcarriers was employed to confirm the advantages of this system, in which high-level expressions of liver-specific functions were observed. Thus, we believe that these cell-laden microcarriers can be used as biological models in organisms and complex multi-tissue studies with the aim of mimicking the behavior of larger organ networks of human bodies.

Received 19 June 2017; accepted 22 July 2017; published online 30 August 2017

1 Souza GR, Molina JR, Raphael RM, et al. Three-dimensional tissue culture based on magnetic cell levitation. Nat Nanotech, 2010, 5: 291-296
2 Sun K, Liu M, Liu H, et al. Semi-egg-like heterogeneous compartmentalization of cells controlled by contact angle hysteresis. Adv Funct Mater, 2015, 25: 4506-4511

3 Yuan B, Jin Y, Sun Y, et al. A strategy for depositing different types of cells in three dimensions to mimic tubular structures in tissues. Adv Mater, 2012, 24: 890-896

4 Lee M, Yang K, Hwang YH, et al. Spheroform: therapeutic spheroid-forming nanotextured surfaces inspired by desert beetle Physosterna cribripes. Adv Healthcare Mater, 2015, 4: 511-515

5 Cheng Y, Zheng F, Lu J, et al. Bioinspired multicompartmental microfibers from microfluidics. Adv Mater, 2014, 26: 5184-5190

$6 \mathrm{Yu} \mathrm{Y,} \mathrm{Fu} \mathrm{F,} \mathrm{Shang} \mathrm{L,} \mathrm{et} \mathrm{al.} \mathrm{Bioinspired} \mathrm{helical} \mathrm{microfibers} \mathrm{from}$ microfluidics. Adv Mater, 2017, 29: 1605765

7 Frey O, Misun PM, Fluri DA, et al. Reconfigurable microfluidic hanging drop network for multi-tissue interaction and analysis. Nat Commun, 2014, 5: 4250

8 Seo J, Lee JS, Lee K, et al. Switchable water-adhesive, superhydrophobic palladium-layered silicon nanowires potentiate the angiogenic efficacy of human stem cell spheroids. Adv Mater, 2014, 26: 7043-7050

9 Chen Y, Shi J. Mesoporous carbon biomaterials. Sci China Mater, 2015, 58: 241-257

10 Cheng Y, Yu Y, Fu F, et al. Controlled fabrication of bioactive microfibers for creating tissue constructs using microfluidic techniques. ACS Appl Mater Interfaces, 2016, 8: 1080-1086

11 Zhang X, Xia LY, Chen X, et al. Hydrogel-based phototherapy for fighting cancer and bacterial infection. Sci China Mater, 2017, 60: 
487-503

12 Zhao Y, Cheng Y, Shang L, et al. Microfluidic synthesis of barcode particles for multiplex assays. Small, 2015, 11: 151-174

13 Liu X, Jin X, Ma PX. Nanofibrous hollow microspheres self-assembled from star-shaped polymers as injectable cell carriers for knee repair. Nat Mater, 2011, 10: 398-406

14 Wang J, Cheng Y, Yu Y, et al. Microfluidic generation of porous microcarriers for three-dimensional cell culture. ACS Appl Mater Interfaces, 2015, 7: 27035-27039

15 Fu F, Shang L, Zheng F, et al. Cells cultured on core-shell photonic crystal barcodes for drug screening. ACS Appl Mater Interfaces, 2016, 8: 13840-13848

16 Maeda $\mathrm{K}$, Onoe $\mathrm{H}$, Takinoue $\mathrm{M}$, et al. Controlled synthesis of 3D multi-compartmental particles with centrifuge-based microdroplet formation from a multi-barrelled capillary. Adv Mater, 2012, 24: 1340-1346

17 Liu Z, Shum HC. Fabrication of uniform multi-compartment particles using microfludic electrospray technology for cell coculture studies. Biomicrofluidics, 2013, 7: 044117

18 Zhao X, Liu S, Yildirimer L, et al. Injectable stem cell-laden photocrosslinkable microspheres fabricated using microfluidics for rapid generation of osteogenic tissue constructs. Adv Funct Mater, 2016, 26: 2809-2819

19 Naqvi SM, Vedicherla S, Gansau J, et al. Living cell factorieselectrosprayed microcapsules and microcarriers for minimally invasive delivery. Adv Mater, 2016, 28: 5662-5671

20 Chen Q, Utech S, Chen D, et al. Controlled assembly of heterotypic cells in a core-shell scaffold: organ in a droplet. Lab Chip, 2016, 16 : 1346-1349

21 Tumarkin E, Kumacheva E. Microfluidic generation of microgels from synthetic and natural polymers. Chem Soc Rev, 2009, 38: 2161-2168

22 Li Y, Yan D, Fu F, et al. Composite core-shell microparticles from microfluidics for synergistic drug delivery. Sci China Mater, 2017, 60: $543-553$

23 Griffin DR, Weaver WM, Scumpia PO, et al. Accelerated wound healing by injectable microporous gel scaffolds assembled from annealed building blocks. Nat Mater, 2015, 14: 737-744

24 Headen DM, Aubry G, Lu H, et al. Microfluidic-based generation of size-controlled, biofunctionalized synthetic polymer microgels for cell encapsulation. Adv Mater, 2014, 26: 3003-3008

25 Shang L, Fu F, Cheng Y, et al. Photonic crystal microbubbles as suspension barcodes. J Am Chem Soc, 2015, 137: 15533-15539

26 Shang L, Fu F, Cheng Y, et al. Bioinspired multifunctional spindle- knotted microfibers from microfluidics. Small, 2017, 13: 1600286

27 Lee TY, Praveenkumar R, Oh YK, et al. Alginate microgels created by selective coalescence between core drops paired with an ultrathin shell. J Mater Chem B, 2016, 4: 3232-3238

28 Sun J, Zhang L, Wang J, et al. Tunable rigidity of (polymeric core)(lipid shell) nanoparticles for regulated cellular uptake. Adv Mater, 2015, 27: 1402-1407

29 Utech S, Prodanovic R, Mao AS, et al. Microfluidic generation of monodisperse, structurally homogeneous alginate microgels for cell encapsulation and 3D cell culture. Adv Healthcare Mater, 2015, 4: 1628-1633

30 Velasco D, Tumarkin E, Kumacheva E. Microfluidic encapsulation of cells in polymer microgels. Small, 2012, 8: 1633-1642

31 Sakai $\mathrm{S}$, Ito $\mathrm{S}$, Inagaki $\mathrm{H}$, et al. Cell-enclosing gelatin-based microcapsule production for tissue engineering using a microfluidic flow-focusing system. Biomicrofluidics, 2011, 5: 013402

32 Matsunaga YT, Morimoto Y, Takeuchi S. Molding cell beads for rapid construction of macroscopic 3D tissue architecture. Adv Mater, 2011, 23: H90-H94

33 Lu YC, Song W, An D, et al. Designing compartmentalized hydrogel microparticles for cell encapsulation and scalable $3 \mathrm{D}$ cell culture. J Mater Chem B, 2015, 3: 353-360

34 Khetani SR, Bhatia SN. Microscale culture of human liver cells for drug development. Nat Biotechnol, 2007, 26: 120-126

Acknowledgements This work was supported by the National Natural Science Foundation of China (21473029 and 51522302), the NSAF Foundation of China (U1530260), the Natural Science Foundation of Jiangsu (BK20140028), the Program for New Century Excellent Talents in University, the Scientific Research Foundation of Southeast University, and the Scientific Research Foundation of Graduate School of Southeast University.

Author contributions Zhao $\mathrm{Y}$ and Wang J conceived the idea and designed the experiment; Wang J carried out the experiments; Wang J and Zhao Y analyzed the data and wrote the paper; Zou M and Sun L assisted with experiment operations. Cheng Y, Shang L and Fu F contributed to scientific discussion of the paper.

Conflict of interest The authors declare that they have no conflict of interest.

Supplementary information online version of the paper. 


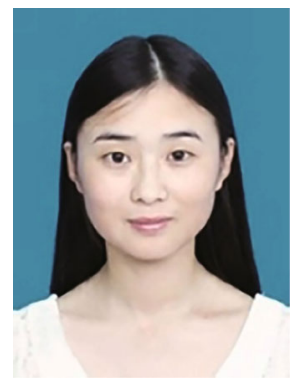

Jie Wang received her BSc degree from Southeast University in 2014. She is now a $\mathrm{PhD}$ candidate under the supervision of Prof. Yuanjin Zhao at Southeast University. Her research interest is in the fabrication of functional materials based on microfluidics.

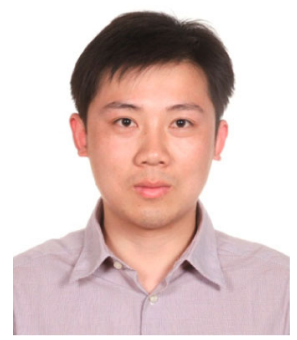

Yuanjin Zhao received his PhD degree in 2011 from Southeast University. In 2009-2010, he worked as a research scholar at Prof. David A. Weitz's group in the School of Engineering and Applied Sciences, Harvard University. Since 2015, he was promoted to be a full professor of Southeast University. His current scientific interests include microfluidic-based materials fabrication, biosensors, and bio-inspired photonic nanomaterials.

\section{微流控制备仿佛珠微载体用于细胞培养研究}

王洁, 邹旻含, 孙灵钰, 程瑶, 商珞然, 付繁繁, 赵远锦“

摘要 构建可用于细胞三维培养的多功能微载体在组织工程的应用中至关重要. 本文受佛珠手串中佛珠可以在绳子上自由滑动这一特殊 结构的启发, 利用毛细管阵列微流控技术制备了一种具有可控大孔微结构的新型异质细胞微载体, 用于细胞三维培养. 仿佛珠微载体的构 建首先需要通过海藻酸钠与钻离子的快速凝胶化形成海藻酸钻纤维, 随即在纤维上包覆可聚合的细胞预聚溶液, 通过流体的剪切实现溶 液乳化并将其固化聚合, 从而获得串有可以自由滑动的微载体的纤维串. 纤维上释放的微载体中间的大孔结构的尺寸高度可控, 这一特点 在微载体用于细胞三维培养中具有重要意义, 因为微载体中间的大孔结构能够有效保证载体内部细胞氧气、营养物质的充分交换, 减少 细胞坏死. 此外, 通过将多种细胞预聚溶液引入微流控通道中, 还可以获得具有多组分异质结构的细胞微载体, 从而有望实现体内复杂的 组织器官结构与功能的模拟. 